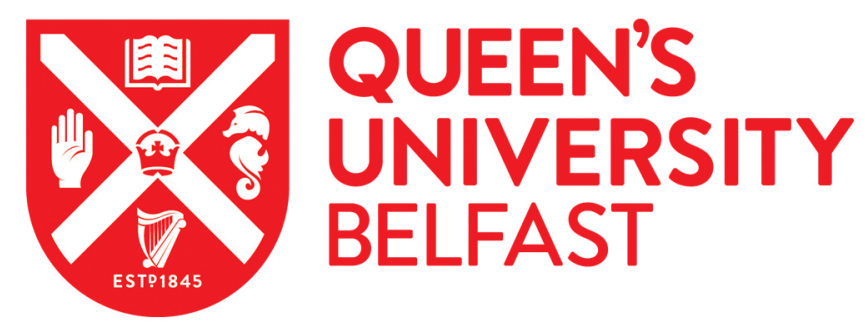

\title{
Predictors of Posttraumatic Stress Symptom Trajectories Following the Fatal 2011 Christchurch, New Zealand Earthquake
}

\author{
Jones, H., Dorahy, M. J., Britt, E., Rowlands, A., Renouf, C., Carter, J. D., \& Hanna, D. (2019). Predictors of \\ Posttraumatic Stress Symptom Trajectories Following the Fatal 2011 Christchurch, New Zealand Earthquake. \\ Journal of Traumatic Stress, 32(2), 206-214. https://doi.org/10.1002/jts.22387
}

Published in:

Journal of Traumatic Stress

Document Version:

Early version, also known as pre-print

Queen's University Belfast - Research Portal:

Link to publication record in Queen's University Belfast Research Portal

Publisher rights

(C) 2019 International Society for Traumatic Stress Studies. This work is made available online in accordance with the publisher's policies. Please refer to any applicable terms of use of the publisher.

\section{General rights}

Copyright for the publications made accessible via the Queen's University Belfast Research Portal is retained by the author(s) and / or other copyright owners and it is a condition of accessing these publications that users recognise and abide by the legal requirements associated with these rights.

Take down policy

The Research Portal is Queen's institutional repository that provides access to Queen's research output. Every effort has been made to ensure that content in the Research Portal does not infringe any person's rights, or applicable UK laws. If you discover content in the Research Portal that you believe breaches copyright or violates any law, please contact openaccess@qub.ac.uk. 
Predictors of Posttraumatic Stress Symptom Trajectories following a fatal earthquake

Hannah Jones, Martin J. Dorahy, Eileen Britt, Amy Rowlands, Charlotte Renouf, and Janet D. Carter

Department of Psychology

University of Canterbury

Christchurch, New Zealand

Donncha Hanna

School of Psychology

The Queen's Univeristy of Belfast

Northern Ireland

Correspondence concerning this article should be addressed to Martin J. Dorahy, Department of Psychology, University of Canterbury, Private Bag 4800, Christchurch, 8140, New Zealand. Email: martin.dorahy@canterbury.ac.nz 


\begin{abstract}
People respond differently to potentially traumatic events. To explore predictors of a chronic and delayed trajectory of posttraumatic stress symptoms after a natural disaster, psychometric data collected from 412 Christchurch residents, post the 2011, 6.3 magnitude earthquake were analysed. Participants from suburbs with different socioeconomic levels and quakes impacts completed a door-to- door survey 4-7 months after the earthquake (time 1, N=600) and again 10-11 months after the quake (time 2, N=412). The survey included the Acute Stress Disorder Scale, the Patient Health Questionnaire's 9-item depression scale and the Generalised Anxiety Disorder -7 scale, along with single item measures of variables like family tension. Hobfoll's Conservation of Resources theory was used to guide data interpretation. High depression (OR: 1.26) and anxiety (OR: 1.25) at time 1 significantly predicted membership to the chronic trajectory. Predictors of a delayed onset of symptoms were increased family tension (OR: 1.37) overtime and living in a low (OR: 4.55) or medium socio-economic area (OR: 11.94). Results highlight risk factors for elevated posttraumatic stress symtoms and resources that can be used by individuals to offset threatened loss. The findings have implications for service providers, agencies and the public.
\end{abstract}


Predictors of Posttraumatic Stress Symptom Trajectories following a fatal earthquake

Posttraumatic stress symptoms (PTSS) develop in a proportion of individuals exposed to a natural disaster (Galea, Nandi \& Vlahov, 2005), with variations attributable to variables such as type and severity of disaster and the assessment of symptoms (Fan et al., 2005). Recent research has explored trajectories of adjustment after potentially traumatic events, using continuous measures of PTSS. Four prototypical trajectories, namely, resilience, recovery, chronic dysfunction and delayed dysfunction, have been supported by empirical evidence to capture most of the variability in trauma response (Bonanno, 2004; Bonanno \& Mancini, 2012; Fan et al., 2015; Tang, 2007). Chronic and delayed trajectories of PTSS are of particular interest to health and social service providers because they characterise the most debilitating trauma outcomes for individuals (Bonanno, 2004). Knowledge of the predictors of these courses would aid in the allocation of often scarce resources to those identified at greatest risk of developing such symptom trajectories (Johanneson, Arinell \& Arnberg, 2015).

The resilient pathway in adults is the most common after exposure to isolated and highly disruptive events, and is a normal human adaptational outcome (Masten, 2001). Bonanno (2004), defines resilience in adults faced with potentially traumatic events, as the ability of individuals "to maintain relatively stable, healthy levels of psychological and physical functioning” (pp. 20-21). Bonanno, Rennick \& Deckel (2005) used consistently low levels of posttraumatic stress and depression as indicators of resilience. Based on cumulative PTSD data collected from participants in New York, post 9/11, Bonanno, Galea, Bucciarelli and Vlahov (2007) found that two thirds of the sample could be reliably characterised as resilient. The finding that $60 \%$ of a sample of Thai survivors of the Southeast Asian earthquake maintained relatively stable functioning is consistent with this finding (Tang, 
2007). Meta analyses show that more than half of the population exposed to a trauma demonstrate resilience (Bonanno \& Mancini, 2012).

In those with elevated initial symptoms, it is usual for functioning to return more or less to baseline after a highly disruptive event (Bonanno \& Mancini 2012). Bonanno (2004) defines recovery in adults exposed to traumatic events as "a trajectory in which normal functioning temporarily gives way to symptoms of PTSD, usually for a period of at least several months, and then gradually returns to pre-event levels" (p. 20). It is also noted, that full recovery may be relatively rapid or it may take as long as one or two years.

Research has shown that as individuals habituated to the 2011 February 22 Christchurch earthquake, distress symptoms reduced (Renouf, 2012). In a longitudinal study on trajectories of posttraumatic stress among adolescents after the Wenchuan earthquake, twenty percent of the sample were classified as being in the recovery group (Fan et al., 2015). This finding is consistent with that of Norris, Tracy and Galea (2009), who found that $19 \%$ of a sample exposed to the 1999 Mexico Floods displayed a trajectory of recovery.

The literature on trajectories of trauma outcomes indicate that between 5 and 20 percent of a population exposed to a disaster will develop high levels of PTSS that persist overtime. These individuals display a trajectory of chronic dysfunction (Bonanno \& Mancini, 2012). After the 2004 Southeast Asian earthquake and tsunami, 12\% of sampled Thai survivors presented with chronic PTSS (Tang, 2007), while $7.2 \%$ of sampled survivors of the 2008 Wenchuan earthquake in China showed chronic PTSS (Fan, Long, Zhou, Zheng \& Liu, 2015). In one study, depression was found to be a significant predictor of chronicity in posttraumatic stress disorder (Freedman, Brandes, Peri \& Shalev, 1999). In another, high baseline levels of anxiety symptoms predicted chronic PTSD in a group of trauma-exposed individuals (Myers, Allan, Korte, Gros \& Acierno, 2017). Furthermore, Fan et al. (2015) 
found that family member loss or injury and low social support 2 years after the Wenchuan earthquake predicted chronic dysfunction.

Although infrequent, delayed reactions to a traumatic event have been empirically verified. Buckley, Blanchard and Hickling (1996) found that some motor vehicle accident survivors did not meet DSM criteria for PTSD 1-4 months after the accident but did after a year. Furthermore, Bonanno, Rennicke and Dekel (2005) found that $13 \%$ of a sample of individuals who had been in or near the World Trade Centre at the time of the 9/11 terrorist attack, had a greater level of PTSD symptoms at 18 months than they did at seven months. Following earthquakes, Tang (2007) found 18\% experienced a delayed onset of dysfunction, with Fan et al. (2015) reporting 4.2\% showing delayed PTSS 2 years after the event. Bonanno and Mancini (2012) concieve delayed PTSS as subthreshold pathology that worsens over time. Significant financial loss, three years after the 1999 earthquake in Taiwan predicted delayed PTSD as did low social support after the 2008 Wenchuan earthquake (Su et al., 2010; Fan et al., 2015).

Based on empirical research, Hobfoll's conservation of resources theory (1989) contends that resource loss underpins stress. Classified as objects, personal characteristics, conditions and energies, resources have both instrumental and symbolic value. According to the model, people strive to retain, protect, and accumulate resources, and it is the potential or actual loss of these resources that is threatening to them. Therefore, psychological distress is conceptualised as a reaction to the environment in which there is, (a) the threat of a net loss of resources, (b) the net loss of resources, or (c) a lack of resource gain following the use of resources. Both perceived and actual loss or lack of gain are sufficient to produce stress.

Object resources, such as a house, are physical in nature and are related to socioeconomic status. Their rarity, expense or personal meaningfulness may add to their value. Conditions, such as marriage, tenure and seniority are resources to the extent that they are 
valued and sought after by an individual. Any given condition may be a resource and therefore protective factor for one person but debilitating for another (Hobfoll, 1989). Personal Characteristics, such as perceiving events as predictable and a positive sense of self are resources in the way that they often mediate resistance to stress (Antonovsky, 1979). The final general form of resources, as proposed by the model, are energies. Examples include time, money and knowledge. It is theorised, that this type of resource is valuable to the extent that it can be used to acquire other resources (Hobfoll, 1989). While not included as its own category, social relations are deemed a resource in so far as they provide or help preserve other valued resources, such as personal characteristics. Research has demonstrated that social support may aid in coping with stressful situations (Cohen \& Wills, 1985).

Unfortunately, resources are not equally distributed. Dohrenwend (1978), contends that individuals who already have a net lack of resources are most vulnerable to additional loss. As the resources to offset loss are not available, a loss spiral may result where one loss perpetuates another (Hobfoll, 1989). The model of conservation of resources (1989) can be applied to research on trauma outcomes. According to the theory, predictors of PTSS should reflect a lack of resources.

On 22 February 2011, a 6.3 magnitude earthquake hit Christchurch, New Zealand. Considered an aftershock of the 7.1 magnitude earthquake that had struck the city in September 2010, 185 lives were lost. Using psychometric data collected from 412 Christchurch residents at two different time points, the current study aimed to explore the predictors of a chronic and delayed trajectory of PTSS after the February 2011 earthquake. These predictors could be targeted by health porofessionals, social service providers and policy makers following natural disasters like earthquakes.

Based on previous research and Hobfoll's (1989) conservation of resources theory, baseline depression and anxiety, and death or injury of someone known were expected to 
predict a chronic trajectory of symptoms. Having to move house, loss of something and living in a suburb more affected by the earthquake were expected as predictors of the delayed trajectory. Increased social isolation and family tension overtime were also expected to predict delayed dysfunction. There is evidence to suggest that a reduction in aftershock anxiety post-earthquake facilitates recovery (Kannis-Dymand et al., 2015). In this way, it is conceivable that increased aftershock anxiety overtime would predict delayed dysfunction. This hypothesis was also tested in the current study. While low socioeconomic status has been found to be correlated with PTSD (Galea, Nandi and Vlahov, 2005), little research has explored it as a predictor of symptom trajectories. It was hypothesised as a predictor of both the delayed and chronic trajectories. As a comparison to the results of the chronic and delayed groups, predictors of the recovery group were also examined.

\section{Method}

\section{Participants}

Participants were 412 residents living in six suburbs in Christchurch, New Zealand. They ranged in age from 18 to 90 years $(M=50.39$ years; $\mathrm{SD}=16.04)$, and the majority were female (65\%). The participants had completed time 1 (baseline) of the study $(\mathrm{N}=600)$ three to six months earlier and gave permission to be followed up (time 2) for further data collection. Suburbs were chosen at the first data collection time point, four-seven months after the 22 February 2011 earthquake, based on the degree of physical damage (either affected or relatively unaffected) caused by the quake and average household income, as drawn from 2006 census data (Statistics New Zealand, 2012). Three of the six suburbs were severely physically affected and comprised low, medium and high average household income areas. Severe physical affectedness was characterised by large scale property and utility loss (see Dorahy et al., 2015, for further details). These three affected suburbs were matched with suburbs that received relatively minimal property and utility loss from the earthquake. 
Suburbs were matched on demographic variables (e.g., population of suburb, average age, sex) and average household income.

\section{Materials}

The questionnaire battery contained the Acute Stress Disorder Scale (ASDS; Bryant, Moulds, \& Guthrie, 2000), the Patient Health Questionnaire's 9-item depression scale (PHQ9; Spitzer, Kroenke, \& Williams, 1999), and the Generalized Anxiety Disorder-7 scale (GAD-7; Spitzer, Kroenke, Williams, \& Lowe, 2006). All items were anchored to symptoms that started since the 22nd February, 2011 earthquake.

The survey began with the ASDS. The PHQ-9, and the GAD-7 followed. After administration of these scales, age and sex were recorded and participants were asked single item questions regarding degree of social isolation, aftershock anxiety and changes in family tension since the February 2011 earthquake. The frequency to which participants had people around to talk to about their experiences since the earthquake was used to measure social isolation. A Likert scale, ranging from 0 (not at all) to 4 (constantly) was used. Ranging from 0 (not at all) to 10 (extremely), participants were asked how anxious the aftershocks had made them feel in the last 3 months. Family tension was measured over the last three months on a scale from 0 (not at all) to 10 (extremely). Participants were also asked questions regarding whether they had to move house, whether they knew someone who had been injured or killed and whether they had lost something (e.g., a house) as a result of the earthquake, on a dichotomous ('yes/no') response scale.

The Acute Stress Disorder Scale (ASDS) contains 19 items based on DSM-IV (American Psychiatric Association, 1994) criteria for acute stress disorder. The scale measures dissociation, re-experiencing, avoidance and arousal on a Likert scale ranging from 1 (not at all) to 5 (very much), and has a minimum score of 19 and the maximum score of 95. 
The ASDS was used in the current study as aftershocks persisted during data collection. The scale is informative about symptoms of posttraumatic stress disorder as it measures the three symptom clusters of DSM-IV PTSD. The current study shows the scale exhibits excellent internal consistency, with Cronbach's $\alpha=>.91$.

The PHQ-9 comprises 9 items from the larger PHQ designed to assess depression. Participants rate the degree to which they have been bothered by each item using a 4-point Likert scale from 0 (not at all) to 3 (nearly every day). Scores range from 0 to 27. The scale has been found to be a reliable and valid measure of depression severity (Kroenke et al., 2010). This is supported by a Cronbach's $\alpha$ of .87 in the current study.

The GAD-7 is a seven-item measure of general anxiety (Spitzer et al., 2006), based on the DSM-IV diagnostic criteria for generalised anxiety disorder. The response format is a 4point Likert scale ranging from 0 (not at all) to 3 (nearly every day). Scores range from 0 to 21. Spitzer et al. (2006) indicate that it is a reliable and valid self-report measure of anxiety. The Cronbach's $\alpha$ in the current study was .91 .

\section{Procedure}

Data were collected via door-to-door surveys. Participants were read aloud each question by the interviewer following the receipt of written informed consent. It took 20-30 minutes to complete the survey. Participants were then debriefed and asked if they would be happy to be followed up in the future. An information sheet detailing free support and counselling services in Christchurch in addition to several self-help educational resources and a $\$ 5$ coffee voucher were given to participants at the end of the survey. Data collection began 4 months after the February $22^{\text {nd }}$ earthquake (time 1 ) and then participants were followed up 10 months after the quake. Data collection took three months at time 1 and two months at time 2. The study was approved by the relevant New Zealand Human Ethics Committee.

\section{Analyses}


The trajectories were derived from the normal distribution of the differences between ASDS scores at time 1 and time 2. As the resilient and chronic trajectories were characterized by little change in symptoms overtime, these two trajectories were made up of those who were within one standard deviation above and below the mean (no change). To diffentiate these two categories, the chronic trajectory, given it reflects persistent high symptom levels, was made up of those participants who were above the mean on the ASDS scale at time 1. The rest of the participants in the first standard deviation of the differences distribution were classed as resilent. As the recovery trajectory was characterized by high symptoms at time 1 and low symptoms at time 2, those above one standard deviation of the mean of the differences distribution (positive scores) were classified as being in this trajectory. The delayed trajectory, characterized by low symptoms at time 1 and increased symptoms at time 2 were classified as those below one standard deviation of the distribution (negative scores).

Scores for family tension, social isolation and aftershock anxiety at time 1 were subtracted from scores at time 2 to produce difference scores. The values $(+/-)$ were then reversed so that an increase in a score reflected an increase in the construct and vice versa.

A univariate analysis of variance (ANOVA) was conducted to test for differences amongst the mean ages of participants across trajectories. The effect size was estimated using partial eta squared. A Games-Howell post hoc test was used to identify differences. The association between sex and trajectories was assessed using a Pearson-Chi Square Test. Finally, to analyse predictors of the chronic, delayed and recovery trajectories, a multinomial logistic regression, in which the resilient group was the reference category was conducted. All analyses were computed using the Statistical Package for the Social Sciences (SPSS Version 22. IBM Corporation, Armonk, NY, USA).

\section{Results}


Table 1 shows the total number, number of male and female (and corresponding percentage), as well as the mean age and standard deviation for each trajectory. A significant difference between the mean age of participants across the trajectories was found, $F(3,406)=3.97, p<.010, \eta_{p}{ }^{2}=.03$. A Games-Howell post-hoc test, showed that the delayed group were older than the chronic, $p=.023$, and recovery, $p=.010$, groups. There was a significant association between sex and trajectory, $\chi^{2}(3)=29.42, p<.001$, with a higher percentage of women in the chronic (79.20\%) and recovery groups $(88.40 \%)$.

Table 2 presents the percentage of participants by trajectory that lived in a suburb more affected by the earthquake, and in a low socio-economic area. A chi-square test showed a significant difference in suburbs across trajectories, $\chi^{2}(15)=49.60, p<.001$. The chronic group had the greatest percentage of participants that lived in a suburb more affected by the earthquake $(71.70 \%)$. The percentage of participants who responded yes to the three dichotomous (yes/no) variables of interest (knew someone killed or injured, having to move house, loss of something) are also given. The association between knowing someone injured or killed as a result of the earthquake and trajectory was non-significant, $\chi^{2}(3)=.61, p=.894$, as was the relationship between losing something as a result of the earthquake and trajectory, $\chi^{2}(3)=6.04, p=.110$. The association between having to move house as a result of the earthquake and trajectory was significant, $\chi^{2}(3)=31.83, p<.001$. The chronic group had the greatest percentage of participants who had to move house as a result of the earthquake $(54.72 \%)$

Table 3 presents the descriptive statistics for the continuous variables across the four trajectories. To test whether depression and anxiety at time 1 would predict trajectory membership, statistics for the PHQ- 9 and GAD-7 were calculated from baseline scores. To test whether change in social isolation, aftershock anxiety and family tension would predict categorical membership, statistics for these variables were derived from the difference 
between scores at time 1 and time 2. The mean PHQ-9 scores for the chronic $(p<.001)$ and recovery $(p<.001)$ groups were significantly higher than those of the other two trajectories, $F(3,408)=68.56, p<.001, \eta_{p}{ }^{2}=.34$. This was the same for the mean GAD-7 sores, $F(3,408)=62.45, p<.001, \eta_{p}{ }^{2}=.32$. Trajectories did not significantly differ in change in social isolation across time, $F(3,406)=.26, p=.854, \eta_{p}{ }^{2}>.00$. A significant difference across trajectories for change in aftershock anxiety overtime was found, $F(3,407)=18.69, p<.001$, $\eta_{p}{ }^{2}=.12$. Games-Howell post-hoc tests showed the only two trajectories that did not differ from one another were the chronic and resilient groups. A significant difference between change in family tension across trajectories was also found, $F(3,406)=12.66, p<.001, \eta_{p}{ }^{2}=.86$. The delayed group differed significantly from all other trajectories $(p s<.05)$. The resilient and recovery groups were also significantly different from one another $(p=.001)$. Between time 1 and 2, family tension and aftershock anxiety generally decreased for participants. The delayed trajectory was the only group, on average, to increase in these constructs overtime.

A multinomial logistic regression was conducted to analyse predictors of the trajectories with the resilient group as the reference group. Level of affectedness of suburbs, death/ injury, social isolation, having to move to a new house, losing something and aftershock anxiety were not found to be significant predictors of either category. Therefore, they were omitted from further analysis. Table 4 shows the results of the regression analysis. A chi-square test of model fit was statistically significant, indicating that the final model reliably predicts categorical membership, $\chi 2(15)=232.98, p<.001$. Nagelkerke's R-Square indicated that $49 \%$ of variance was explained by the predictors included in the final model.

Consistent with the hypotheses, depression and anxiety at baseline significantly predicted whether an individual was in the chronic or resilient group. As depression scores increased by one unit, the change in the odds of being in the chronic group compared to the 
resilient group was 1.26 . The change in the odds of being in the chronic group compared to the resilient group was 1.25 as anxiety increased by one unit.

Increased family tension overtime and living in a low socioeconomic area predicted membership to the delayed trajectory. As family tension scores increased by one unit, the change in the odds of being in the delayed group compared to the resilient group was 1.37. The odds of being in the delayed group compared to the resilient group changed by 4.55 units as the socioeconomic status of a suburb changed from high to low. As the socioeconomic status of an area changed from high to medium, the odds of being in the delayed group compared to the resilient group changed by 11.94 units. Sixty seven percent of people in the delayed group lived in medium socioeconomic areas $(26 \%$ in low, and $7 \%$ in high socioeconomic areas).

The analysis revealed that depression and anxiety at time 1 significantly predicted whether an individual was in the recovery group or the resilient group. For every one unit increase in depression at baseline, the odds of being in the recovery group compared to the resilient group changed by 1.24 units. Likewise, the odds of being in the recovery group instead of the resilient group changed by 1.29 units for every one unit increase in anxiety at time 1 . Change in family tension overtime also significantly predicted whether an individual was in the recovery or the resilient group. A decrease in family tension between the two time points predicted membership to the recovery group. Finally, compared to those living in a high socio-economic area, residents of a low socio-economic area were 3.56 times less likely to be in the recovery group than the resilient group.

\section{Discussion}

The primary aim of the study was to analyse predictors of the chronic and delayed trajectories of posttraumatic stress symptoms after a natural disaster. Consistent with the hypotheses, depression and anxiety at baseline were significant predictors of the chronic trajectory. The 
findings that increasing family tension overtime and living in a low socio-economic area were predictors of membership to the delayed trajectory also support the hypotheses. While social isolation was not found to be a significant predictor of categorical membership, as discussed below low social support may be best captured in the family tension variable. Unexpectedly, living in a medium socio-economic area was also found to be a significant predictor of membership to the delayed group. High depression and anxiety at baseline, decreased family tension overtime and living in a high socio-economic area were found to predict membership to the recovery group compared to the resilient group.

The finding that depression and anxiety at time 1 predicted a trajectory of chronic dysfunction suggests that those who possess these particular symptoms in the early aftermath of a natural disaster are at a greater risk of maintaining elevated levels of PTSS. It is well established that anxiety, depression and posttraumatic stress often co-exist. Those with PTSD are 3-5 times more likely to also have depression than those that do not have the disorder, and anxiety constitutes one of the characteristic features of PTSD (Kessler, Sonnega, Bromet, Hughes \& Nelson, 1995; Zoladz, Fleshner \& Diamond, 2012).

That depression and anxiety also predicted the recovery trajectory implies that these symptoms are predictive of elevated levels of posttraumatic stress after a traumatic event in general rather than the trajectories themselves. While the odds of developing chronic dysfunction are increased, some individuals who possess these symptoms in the initial aftermath of a traumatic event will go on to recover. This demonstrates the variability in trauma response and that a percentage of people will recover from early heightened symptoms.

While currently there is a lack of research on the effect of socioeconomic status on trajectory membership after a traumatic event, Hobfoll's conservation of resources theory (1989) corroborates the finding that living in a low socioeconomic area predicts delayed 
dysfunction. The model proposes that socioeconomic status is an important factor involved in stress resistance. Low household income represents the lack of an 'energy' resource, which would have otherwise been used to offset loss after a stressful event. The other predictor variables of both the delayed and chronic trajectories also support this theory. According to the model, family tension is construed as the lack of a 'social' resource and depression and anxiety represent the lack of 'personal' resources. Hobfoll (1989) contends that if these resources had been present they would have been used to offset loss. Instead, individuals with a lack of resources are more vulnerable to additional loss, hence elevated posttraumatic stress symptoms (Dohrenwend, 1978).

Decreasing family tension overtime (which may also mean increased social support) appears to have helped people recover instead of maintaining elevated levels of stress. This suggests that one of the factors that differentiates between the chronic and recovery trajectories is the level of family tension. Change in family tension overtime predicted both the recovery and the delayed trajectory. Reductions in family tension predicted recovery and increases in family tension predicted a delayed response. This is consistent with the finding that after the 2008 Wenchuan earthquake, low social support predicted a delayed onset of PTSS (Fan et al., 2015).

Those living in a low socioeconomic area were less likely than those living in a high socioeconomic area to be in the recovery group. This suggests that with low household income, the lack of an 'energy' resource, may have prevented individuals from returning to reduced levels of PTSS (Hobfoll, 1989). This finding doesn't corroborate with the results of the chronic trajectory however, which did not find socioeconomic status to be a significant predictor of chronic dysfunction. Thus, while socioeconomic status does not predict posttraumatic stress chronicity at a statistically significant level, low socioeconomic status appears to be a risk factor for a failure to recover from posttraumatic stress symptoms, 
perhaps most particularly in those who did not meet the severity threshold for the chronic trajectory. Such an interpretation aligns with the conservation of resources theory which predicts that those living in a high socio-economic area possess the resources to offset loss, even if initially stress is induced (Hobfoll, 1989).

Despite socioeconomic status being a significant predictor of the delayed trajectory, none of the variables indicative of financial loss were. This is contrary to the hypotheses, and findings of Su et al. (2010). Regardless of the percentage of participants in the chronic group that lived in a suburb more affected by the earthquake, suburb affectedness was not a significant predictor of this trajectory either. These results may have to do with the validity of the items as measures of financial loss or the magnitude of the Christchurch Earthquake compared to other disasters. Financial loss may be particularly predictive of post-traumatic stress in the aftermath of larger more destructive disasters (Su et al., 2010).

Despite $42 \%$ of the chronic group knowing someone who had been killed or injured as a result of the earthquake, this variable was not a significant predictor of the trajectory, unlike the findings of Fan et al. (2015). Again, this may be attributable to the severity and scale of the disaster compared to others. While, on average, aftershock anxiety increased overtime for those in the delayed group, this was not a statistically significant predictor of membership to this trajectory.

The odds of being in the delayed group compared to the resilient group were even greater for those in a medium socio-economic area (when compared to high) than for those in a low socio-economic area. With the conservation of resources theory proposing that household income acts as a resource to offset loss threatened by a stressful event, those living in a medium socio-economic area may use this available resource in the immediate aftermath of an event. But such a resource is limited and the model contends that environmental circumstances threaten or cause depletion of resources (Hobfoll, 1989). While those with 
moderate household income may initially be able to offset threatened loss, resources exist to be used and can therefore diminish. Diminished resources may explain why individuals living in a medium socio-economic area, are at a greater risk of being in the delayed group than those living in a low socio-economic area. Eventually, resources deplete, and individuals who initially cope well are unable to continue offsetting loss and therefore stress, hence a delayed dysfunction trajectory of PTSS. The way in which those living in a high socioeconomic area are the least likely to be in the delayed group supports this theory further. According to the conservation of resources theory, they are likely to have the most 'energies' resource due to their relative wealth. This resource is a protective factor against immediate or delayed dysfunction.

Knowing that depression and anxiety at baseline are significant predictors of a chronic trajectory of PTSS is important for health and social service providers. Treatment of those presenting with these symptoms in the aftermath of a potentially traumatic event should be prioritised. By doing this, personal resources that can be used to offset future loss will be restored (Hobfoll, 1989).

It is important for service providers, governmental and non-governmental agencies, funding bodies and the public to be aware, that the level of family tension can influence individual PTSS trajectories. The results clearly show that decreased family tension after a period of stress can lead to a reduction in symptoms and increased family tension after a period of no symptoms is predictive of delayed elevated levels of post-traumatic stress. This demonstrates the power of family cohesion and thus social support amid destabilising times. Social relations are a resource that can be used when the threat of loss is imminent. The diminishing of social support can lead to negative outcomes for people, whereas the replenishing or replacement of it can lead to better outcomes for people (i.e., recovery). 
The knowledge that living in a low socio-economic area predicts the delayed trajectory and that this group is less likely to be in the recovery trajectory is another risk factor that health professionals should be aware of. Those with a lower socioeconomic status need more support in the aftermath of a natural disaster (Dorahy et al., 2015).

The finding that medium socio-economic status also predicts the delayed trajectory suggests that people need a surplus and variety of resources. It appears that having a moderate household income may not be sufficient to buffer against the impact of a potentially traumatic event. Different kinds of resources are important in different ways and can all be used to offset loss threatened by a stressful event. Resources can also be accumulated and replenished even after the event has occurred (Hobfoll, 1989). An example of this from the current study was a decrease in family tension, corresponding to an increase in social support. This was predictive of recovery rather than the continuation of elevated posttraumatic stress symptoms. In the aftermath of a natural disaster, individuals, families and communities should be intentional about accumulating additional resources. This may prevent the development of a delayed stress reaction.

A limitation of this study is that data collection only occurred at two time points and the window between these ranged from 3-7 months in length. Previous research on symptom trajectories have collected data in 6 month intervals to allow enough time for change in symptoms to occur (Fan et al., 2015). The confidence to claim that participants are displaying trajectories of posttraumatic stress symptoms would be strengthened if data were collected at three or more time points with at least a 6-month gap between.

Future work should tease apart the variables that determine if a person remains in the chronic group or moves to the recovery group, as depression and anxiety at time 1 were elevated in both trajectories. The current study aimed to examine predictors of chronic and delayed trajectories of posttraumatic stress symptoms after a natural disaster. Baseline 
depression and anxiety were found to predict chronic dysfunction and increased family tension, and living in a low or medium socioeconomic area predicted a delayed trajectory. The results corroborate Hobfoll's conservation of resources theory (1989), which asserts that a lack of resources makes people vulnerable to further loss and therefore stress. The results of this study would be helpful to health and social service providers, policy makers, and the public. By knowing the predictors of chronic and delayed trajectories of PTSS, mental health professionals are enabled to prioritise individuals most at risk for support and treatment and policy makers can most effectively deliver resources to maximize recovery. Furthermore, the current study demonstrates the power of resources. Even amidst stress, resources, such as family cohesion or social support, can be replenished or accumulated. These can be used to offset further loss and have the capacity to bring about recovery. 


\section{References}

American Psychiatric Association (1994). Diagnostic and Statistical Manual of Mental Disorders (4th ed.) Washington, DC: Author.

American Psychiatric Association (2013). Diagnostic and Statistical Manual of Mental Disorders: (5 $5^{\text {th }}$ ed.) Washington, DC: Author.

Antonovsky, A. (1980). Health, stress, and coping (1st ed.). San Francisco: Jossey-Bass Publishers.

Buckley, T. C., Blanchard, E. B., \& Hickling, E. J. (1996). A prospective examination of delayed onset PTSD secondary to motor vehicle accidents. Journal of Abnormal Psychology, 105(4), 617-625. doi:10.1037/0021-843X.105.4.617

Bonanno, G. A. (2004). Loss, trauma, and human resilience: have we underestimated the human capacity to thrive after extremely aversive events? American Psychologist, 59(1), 20-28. doi: 10.1037/0003-066X.59.1.20

Bonanno, G. A., Galea, S., Bucciarelli, A., \& Vlahov, D. (2007). What predicts psychological resilience after disaster? The role of demographics, resources, and life stress. Journal of Consulting and Clinical Psychology, 75(5), 671-682. doi:10.1037/0022006X.75.5.671

Bonanno, G. A., \& Mancini, A. D. (2012). Beyond resilience and PTSD: Mapping the heterogeneity of responses to potential trauma. Psychological Trauma: Theory, Research, Practice, and Policy, 4(1), 74-83. doi:10.1037/a0017829

Bonanno, G. A., Rennicke, C., \& Dekel, S. (2005). Self-enhancement among high-exposure survivors of the September 11th terrorist attack: Resilience or social maladjustment? Journal of Personality and Social Psychology, 88(6), 984-998. doi:10.1037/00223514.88.6.984 
Bryant, R. A., \& Harvey, A. G. (2000). Acute stress disorder: A handbook of theory, assessment, and treatment. Washington, DC: American Psychological Association.

Cohen, S., \& Wills, T. A. (1985). Stress, social support, and the buffering hypothesis. Psychological Bulletin, 98(2), 310-357. doi: 10.1037/0033-2909.98.2.310

Dohrenwend, B. S. (1978). Social stress and community psychology. American Journal of Community Psychology, 6(1), 1-14. doi: 10.1007/BF00890095

Dorahy, M. J., Rowlands, A., Renouf, C., Hanna, D., Britt, E., \& Carter, J. D. (2015). Impact of average household income and damage exposure on post-earthquake distress and functioning: A community study following the February 2011 Christchurch earthquake. British Journal of Psychology, 106(3), 526-543. doi:10.1111/bjop.12097

Fan, F., Long, K., Zhou, Y., Zheng, Y., \& Liu, X. (2015). Longitudinal trajectories of posttraumatic stress disorder symptoms among adolescents after the Wenchuan earthquake in China. Psychological Medicine, 45(13), 2885-2896. doi: $10.1017 / \mathrm{S} 0033291715000884$

Freedman, S. A., Brandes, D., Peri, T., \& Shalev, A. (1999). Predictors of chronic posttraumatic stress disorder. A prospective study. The British Journal of Psychiatry, 174(4), 353-359. doi: 10.1192/bjp.174.4.353

Galea, S., Nandi, A., \& Vlahov, D. (2005). The epidemiology of post-traumatic stress disorder after disasters. Epidemiologic Reviews, 27(1), 78-91. doi: 10.1093/epirev/mxi003

Hobfoll, S. E. (1989). Conservation of Resources: A New Attempt at Conceptualizing Stress. American Psychologist, 44(3), 513-524. doi:10.1037/0003-066X.44.3.513

Johannesson, K. B., Arinell, H., \& Arnberg, F. K. (2015). Six years after the wave. Trajectories of posttraumatic stress following a natural disaster. Journal of Anxiety Disorders, 36, 15-24. doi: 10.1016/j.janxdis.2015.07.007 
Kannis-Dymand, L., Dorahy, M. J., Crake, R., Gibbon, P., \& Luckey, R. (2015). An exploration of reported cognitions during an earthquake and its aftershocks:

Differences across affected communities and associations with psychological distress. The Journal of Nervous and Mental Disease, 203(4), 279-286. doi: 10.1097/NMD.0000000000000282

Kessler, R. C., Sonnega, A., Bromet, E., Hughes, M., \& Nelson, C. B. (1995). Posttraumatic stress disorder in the National Comorbidity Survey. Archives of General Psychiatry, 52(12), 1048-1060. doi:10.1001/archpsyc.1995.03950240066012

Kroenke, K., Spitzer, R. L., Williams, J. B., \& Lowe, B. (2010). The Patient Health Questionnaire $€$ somatic, anxiety, and depressive symptom scales: A systematic review. General Hospital Psychiatry, 32, 345-359. doi:10.1016/j.genhosppsych.2010.03.006

Masten, A. S. (2001). Ordinary magic: Resilience processes in development. American Psychologist, 56(3), 227-238. doi: 10.1037/0003-066X.56.3.227

Myers, U. S., Allan, N. P., Korte, K. J., Gros, D. F., \& Acierno, R. (2017). Predictors and outcomes of growth mixture modeled trajectories across an exposure-based PTSD intervention with veterans. Journal of Clinical Psychology, 73(9), 1048-1063 doi:10.1002/jclp.22408

Norris, F. H., Tracy, M., \& Galea, S. (2009). Looking for resilience: Understanding the longitudinal trajectories of responses to stress. Social Science \& Medicine, 68(12), 2190-2198. doi: 10.1016/j.socscimed.2009.03.043

Renouf, C. A. (2012). Do the psychological effects of ongoing adversity in a natural context accumulate or lessen over time? The case of the Canterbury earthquakes. University of Canterbury, 1-97. Retreieved from: https://ir.canterbury.ac.nz 
Spitzer, R., Kroenke, K., \& Williams, J. B. W. (1999). Validation and utility of a self- report version of PRIME-MD: The primary care study. Journal of the American Medical Association, 282, 1737- 1744. doi:10.1001/jama.282.18.1737

Spitzer, R., Kroenke, K., Williams, J. B. W., \& Lowe, B. (2006). A brief measure for assessing $€$ generaliszed anxiety disorder. Archives of Internal Medicine, 166, 10921097. doi:10.1001/ archinte.166.10.1092

Statistics New Zealand (2012). 2006 Census. Retrieved from http://www.stats.govt.nz/Census/ 2006CensusHomePage.aspx.

Su, C. Y., Tsai, K. Y., Chou, F. H. C., Ho, W. W., Liu, R., \& Lin, W. K. (2010). A three-year follow-up study of the psychosocial predictors of delayed and unresolved posttraumatic stress disorder in Taiwan Chi-Chi earthquake survivors. Psychiatry and Clinical Neurosciences, 64(3), 239-248. doi: 10.1111/j.1440-1819.2010.02087.x

Tang, C. S.-k. (2007). Trajectory of Traumatic Stress Symptoms in the Aftermath of Extreme Natural Disaster: A Study of Adult Thai Survivors of the 2004 Southeast Asian Earthquake and Tsunami. The Journal of Nervous and Mental Disease, 195(1), 54-59. doi:10.1097/01.nmd.0000242971.84798.bc

Zoladz, P. R., Fleshner, M., \& Diamond, D. M. (2012). Psychosocial animal model of PTSD produces a long-lasting traumatic memory, an increase in general anxiety and PTSDlike glucocorticoid abnormalities. Psychoneuroendocrinology, 37(9), 1531-1545. doi:10.1016/j.psyneuen.2012.02.007 
Table 1

Demographic Variables by Post-Traumatic Stress Trajectory

Trajectory Total Number Mean Age(SD) Number of Male(\%) Number of Female(\%)

\begin{tabular}{lcccc}
\hline Chronic & 53 & $47.21(14.25)$ & $11(20.80)$ & $42(79.20)$ \\
Resilient & 247 & $51.00(16.79)$ & $102(41.30)$ & $145(58.70)$ \\
Recovery & 69 & $46.94(13.21)$ & $8(11.60)$ & $61(88.40)$ \\
Delayed & 43 & $56.40(16.14)$ & $21(48.80)$ & $22(51.20)$ \\
\hline
\end{tabular}


Table 2

Percentage of Participants in Each Nominal Variable as a Function of PTSS Trajectory

Suburb more $\quad$ Low SES Death/Injury Move House Loss

Affected

\begin{tabular}{|c|c|c|c|c|c|}
\hline $\begin{array}{l}\text { Total expected } \\
\text { in each group }\end{array}$ & 50.00 & 33.33 & 41.91 & 31.22 & 25.50 \\
\hline Chronic & 71.70 & 33.97 & 41.51 & 54.72 & 35.85 \\
\hline Resilient & 41.30 & 32.80 & 43.21 & 23.27 & 25.10 \\
\hline Recovery & 53.62 & 34.78 & 40.58 & 47.83 & 26.09 \\
\hline Delayed & 44.19 & 25.58 & 37.21 & 20.93 & 13.95 \\
\hline
\end{tabular}


Table 3

Mean (SD) for Distress Measures and Additional Items as a Function of PTSS Trajectory

\begin{tabular}{lccccc}
\hline & PHQ-9 & GAD-7 & Family & Social & Aftershock \\
& & & Tension & Isolation & Anxiety \\
\hline Chronic & $9.94(5.12)$ & $9.55(4.12)$ & $-1.27(2.70)$ & $-.06(1.20)$ & $-.92(3.71)$ \\
Resilient & $3.57(3.17)$ & $3.98(3.34)$ & $-.65(2.23)$ & $-.07(.90)$ & $-.93(2.17)$ \\
Recovery & $9.64(5.36)$ & $9.38(4.61)$ & $-1.96(2.52)$ & $-.19(1.27)$ & $-2.55(2.58)$ \\
Delayed & $3.47(4.36)$ & $3.53(3.96)$ & $.74(2.26)$ & $-.12(1.49)$ & $.86(2.56)$ \\
\hline
\end{tabular}

Note. $\mathrm{CI}=$ confidence interval. 
Table 4

Output from the Multinomial Logistic Regression Analysis that Predicted Trajectories from Family Tension, Depression, Anxiety and Socioeconomic Status of Suburb

\begin{tabular}{lccc}
\hline & \multicolumn{3}{c}{$95 \%$ CI for Odds } \\
& Ratio & \\
& & Odds Ratio & Upper \\
\hline
\end{tabular}

Chronic vs. Resilient

$\begin{array}{llccc}\text { Family Tension } & -0.06(0.08) & .817 & .95 & 1.10 \\ \text { Depression } & 0.23(0.05)^{* * *} & 1.14 & 1.26 & 1.39 \\ \text { Anxiety } & 0.22(0.05)^{* * *} & 1.12 & 1.25 & 1.40 \\ \text { Low SES } & -0.65(0.48) & .20 & .53 & 1.35 \\ \text { Medium SES } & 0.33(0.47) & .56 & 1.40 & 3.51\end{array}$

Recovered vs.

Resilient

\begin{tabular}{llrrr} 
Family Tension & $-0.18(0.07)^{*}$ & .73 & .84 & .96 \\
Depression & $0.22(0.05)^{* * *}$ & 1.14 & 1.24 & 1.36 \\
Anxiety & $0.23(0.05)^{* * *}$ & 1.13 & 1.29 & 1.40 \\
Low SES & $-1.27(0.42)^{* *}$ & .12 & .28 & .64 \\
Medium SES & $-0.68(0.44)$ & .22 & .51 & 1.19 \\
\hline Delayed vs. & &. & & \\
Resilient & & & & \\
& & & & \\
FamilyTension & $0.31(0.08)^{* * *}$ & 1.16 & 1.37 & 1.61 \\
Depression & $0.02(0.07)$ & .88 & 1.02 & 1.17 \\
Anxiety & $-0.00(0.08)$ & .85 & 1.00 & 17.92 \\
Low SES & $1.52(0.70)^{*}$ & 1.16 & 4.55 &
\end{tabular}


$\begin{array}{lllll}\text { Medium SES } & 2.48(0.64)^{* * *} & 3.41 & 11.94 & 41.85\end{array}$

Note. $\mathrm{R} 2=.43$ (Cox \&Snell), .49 (Nagelkerke). Model $\chi 2(15)=232.98, p<.001 .{ }^{*} p<.05,{ }^{* *} p<.01,{ }^{* * *} p<.001$ 